\title{
Endovascular management of pelvic trauma
}

\author{
Arlene Weir ${ }^{1,2}$, Padraic Kennedy ${ }^{1,2}$, Stella Joyce ${ }^{1,2,3}$, David Ryan ${ }^{3}$, Liam Spence ${ }^{1}$, Mark McEntee ${ }^{3}$, \\ Michael Maher ${ }^{1,2,3}$, Owen O'Connor ${ }^{1,2,3}$
}

${ }^{1}$ Department of Radiology, Cork University Hospital, Wilton, Cork, Ireland; ${ }^{2}$ Department of Radiology, Mercy University Hospital, Grenville Place, Cork, Ireland; ${ }^{3}$ School of Medicine, University College Cork, Cork, Ireland

Contributions: (I) Conception and design: A Weir, P Kennedy, O O’Connor; (II) Administrative support: S Joyce, D Ryan, L Spence, M Maher; (III) Provision of study materials or patients: L Spence, M Maher, O O'Connor; (IV) Collection and assembly of data: A Weir, P Kennedy, S Joyce, O

O'Connor; (V) Data analysis and interpretation: A Weir, P Kennedy, O O'Connor; (VI) Manuscript writing: All authors; (VII) Final approval of manuscript: All authors.

Correspondence to: Dr. Arlene Weir. Postal address: Department of Radiology, Cork University Hospital, Wilton, Cork, Ireland. Email: arlene.weir@hse.ie.

\begin{abstract}
Traumatic pelvic injuries are an important group of acquired pathologies given their frequent association with significant vascular compromise. Potentially fatal as a consequence of rapid hemorrhage, achievement of early hemostasis is a priority; endovascular management of traumatic pelvic arterial injuries is an important potential option for treatment. Precipitated by any number of mechanisms of trauma, pelvic vascular injury necessitates timely patient assessment. Variable patterns of arterial injury may result from blunt, penetrating or iatrogenic trauma. Selection of the most appropriate imaging modality is a priority, ensuring streamlined access to treatment. In the case of CT, this is complemented by acquisition of the most appropriate phase of imaging; review of both arterial and delayed phase imaging improves the accuracy of detection of low-flow hemorrhage. In cases where surgical intervention is not deemed appropriate, endovascular treatment provides an alternative means for cessation of hemorrhage associated with pelvic injuries. This may be achieved in a selective or nonselective manner depending on the patient's clinical status and time constraints. Consequently, a detailed understanding of vascular anatomy is essential, including an appreciation of the normal variant anatomy between males and females. Additional consideration must be given to variant anatomy which may co-exist in both sexes. This review article aims to provide a synopsis of endovascular management of pelvic vascular injury. Through case examples, available treatment options will be discussed, including thrombin injection and transcatheter arterial embolization. Furthermore, potential adverse complications of pelvic arterial embolization will be highlighted. Finally, in view of the potential severity of these injuries, a brief overview of initial management of the hemodynamically unstable patient is provided.
\end{abstract}

Keywords: Pelvic trauma; vascular injury; endovascular; interventional radiology

Submitted Jun 09, 2020. Accepted for publication Dec 18, 2020.

doi: 10.21037/atm-20-4591

View this article at: http://dx.doi.org/10.21037/atm-20-4591

\section{Introduction}

Pelvic trauma is a broad term consisting of injuries which may be incurred through many different etiologies, including iatrogenic injury, low and high velocity penetrating trauma, and blunt injury. It is widely accepted that pelvic trauma is associated with a high degree of morbidity and mortality, primarily due to vascular injury $(1,2)$.

The pelvis is a rigid structure containing a number of large caliber vessels, with injury being potentially catastrophic due to the combination of rapid hemorrhage and difficulty obtaining hemostasis (3). Historically, endovascular intervention was not considered to be part of first line management of hemorrhage in pelvic trauma, however, endovascular management of pelvic trauma has gained widespread acceptance in this setting in the advent of improved imaging, clinical skills and experience. Endovascular intervention can prevent significant morbidity associated with surgery and can quickly access and control sites 
of hemorrhage (4), which has led to it becoming the first line method for the treatment of arterial injury caused by pelvic trauma (5).

In an unstable patient, where a pelvic source of hemorrhage is clinically suspected it is reasonable to proceed directly to direct angiography and endovascular management of a bleeding vessel if direct surgical intervention is not deemed appropriate. It has previously been reported that $12-15 \%$ of patients die from an associated injury (6) and surgical exploration of vascular trauma may divert the surgeon's attention from other injuries, decompress hematoma and increase blood loss in the pelvic space which is very confined. In addition to this, surgeons may only directly visualize the exterior of a vessel and injuries such as intimal damage may be missed. Surgical intervention carries significant risk associated with general anesthesia, disruption of a hematoma and infection (7). It should be noted, however, that procedural delays can occur due to the time required for specialized staff to arrive, determining the location of vascular injury and obtaining endovascular access. Therefore, endovascular management should only be considered in the unstable patient when there will be no excessive delay to management or when other steps have been taken to slow the rate of bleeding including pelvic packing, aortic balloon occlusion and/or blood transfusions as deemed necessary (3).

In a hemodynamically stable patient CT is a useful method for the detection of the site of hemorrhage. Three phase imaging is the CT examination of choice in this scenario with non-contrast imaging providing reference for post contrast phases which can help differentiate calcification from hemorrhage on contrast enhanced imaging. Arterial phase imaging is most likely to show a vascular blush or active hemorrhage and delayed phase imaging provides a second opportunity to demonstrate low flow hemorrhage or contrast pooling. It is noted, however, that not all patients with contrast blush on CT require endovascular management and conversely some patients without blush being identified on CT may still require endovascular intervention if clinically indicated (8). This paper will discuss different types of vascular injury and their etiologies, while examining different imaging techniques and pathways for management of pelvic vascular injury.

\section{Etiology}

\section{Iatrogenic Injury}

Iatrogenic injury to pelvic vessels is a relatively uncommon but serious risk of surgical and endovascular intervention. There are a wide variety of iatrogenic injuries which can occur during surgical intervention. For example, vascular injury most commonly occurs during laparoscopic surgery when introducing a Veress needle or primary trocar, with these being implicated with injury to distal aorta or iliac vessels (9) (Figure 1) particularly during the early proliferation of laparoscopic surgery prior to the development of access devices which provide protection against vascular or bowel injury. Rates of vascular injury during laparoscopy are relatively uncommon, ranging from $0.01-0.64 \%$ (10), however, mortality rates can be as high as 15 percent (11). Open abdominal or pelvic surgeries generally allow for direct visualization and management of vascular injury if this should occur, however endovascular management can play a role in the postoperative patient showing signs of persistent blood loss whose hemodynamic status will allow for endovascular management over surgical re-exploration (12). Other causes of iatrogenic arterial hemorrhage also include injury to the abdominal wall or pelvic viscera from intervention such as the excision of endometriomas, peritoneal deposits or following cesarean section and other intrauterine interventions including evacuation of retained products of conception (Figure 2). Studies have shown that up to $18 \%$ of retained products of conception have marked vascularity, resulting in increased risk of hemorrhage during surgical intervention (13). Some institutions use uterine artery embolization as an adjunct in the management of retained products of conception (14) or following prolonged post-partum hemorrhage when management with conservative measures has failed (15). Iatrogenic injury may also occur at the site of vascular access or bypass anastomosis, with pseudoaneurysms being reported as a complication of $1.4-4 \%$ of arterial anastomoses (Figure 3) (16).

\section{Penetrating trauma}

Pelvic arterial injury may result from penetrating injuries such as gunshot wounds, stab wounds and impalement. Penetrating trauma is typically associated with mural laceration, complete or incomplete transection and arteriovenous fistula formation (17). Hemorrhage is the most common result of penetrating trauma and in many circumstances endovascular intervention is deemed the most appropriate management, however, the use of CT or endovascular imaging for initial assessment is usually determined by the patient's hemodynamic status. 

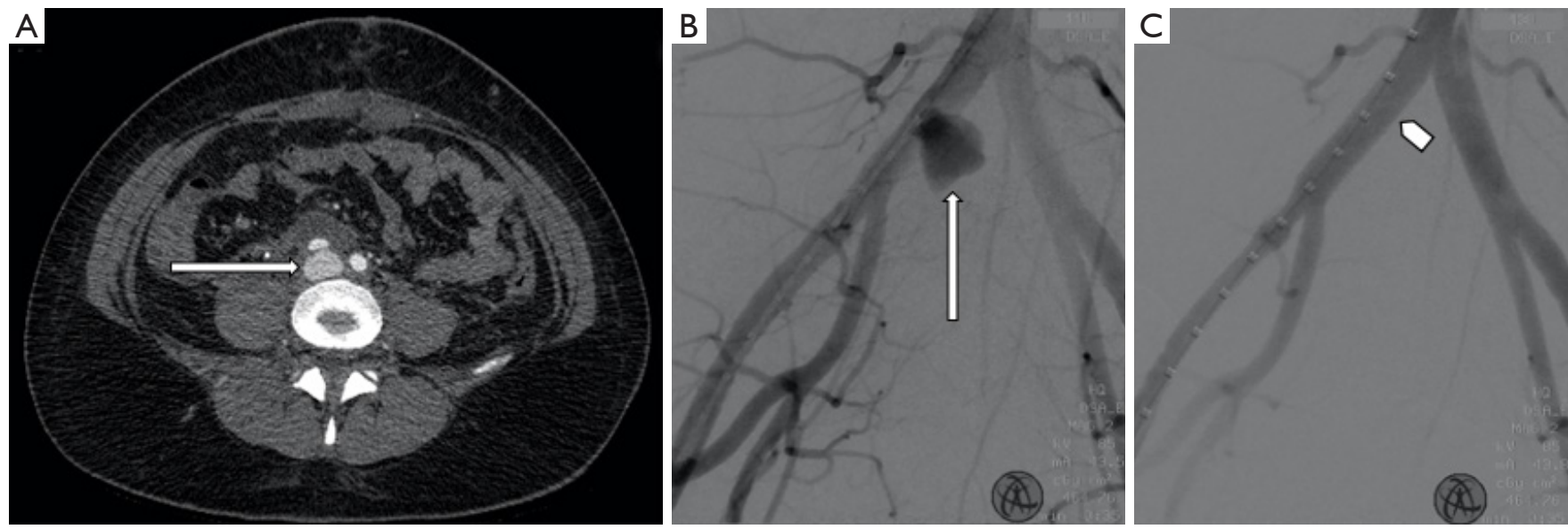

Figure 1 A 39-year-old female who underwent laparoscopy, that was complicated by a vascular tear which was repaired intraoperatively. (A) Subsequent contrast enhanced CT angiography demonstrates a pseudoaneurysm arising from the right common iliac artery posteriorly (long arrows), with adjacent hematoma. (B) The patient subsequently underwent transcatheter angiography. (C) Placement of a selfexpanding covered stent and occlusion of the pseudoaneurysm (pentagon arrow).
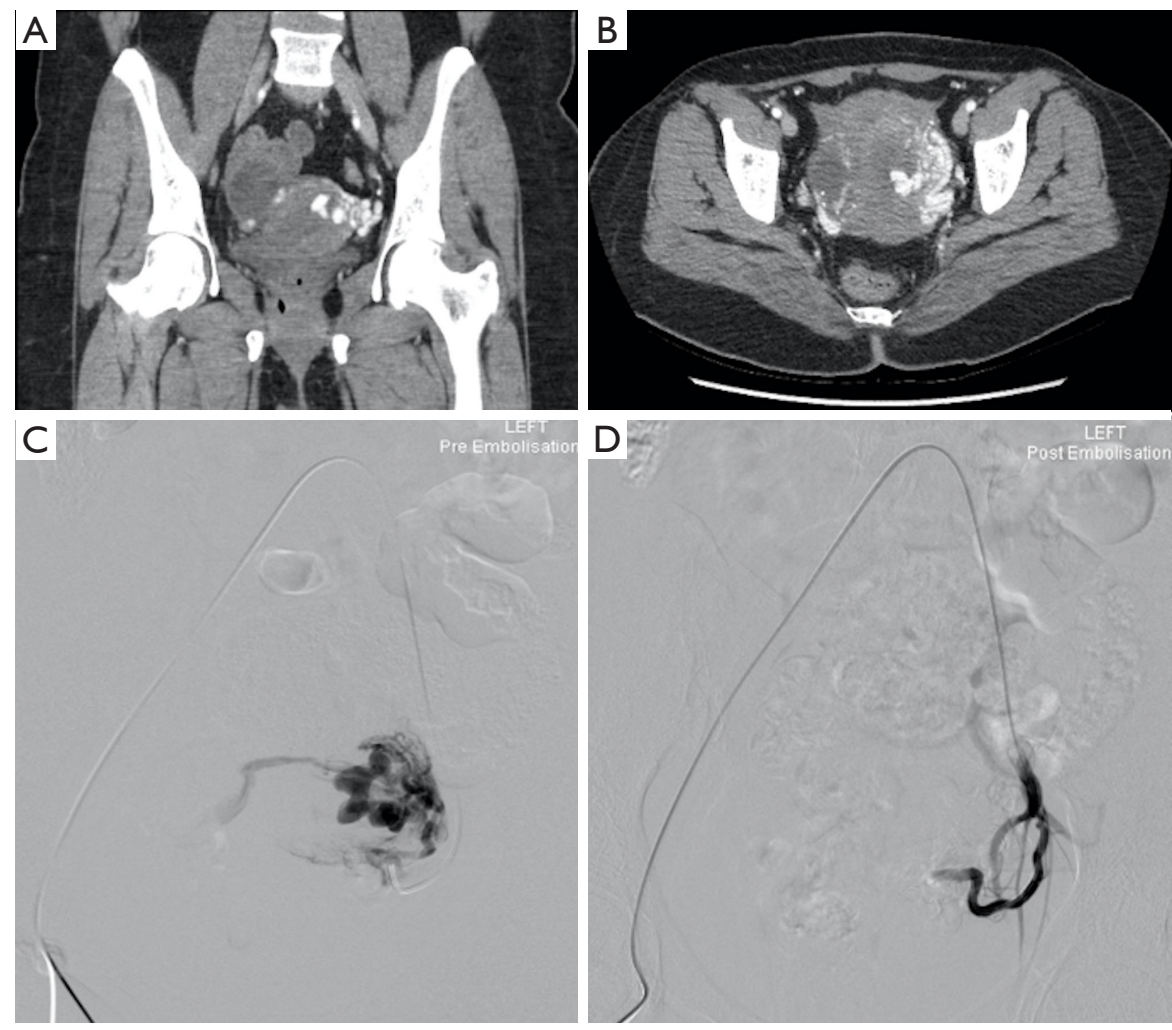

Figure 2 A 31-year-old female who suffered a miscarriage at ten weeks gestation and underwent an evacuation of retained products of conception (ERPC) with two liters of blood loss intraoperatively. The patient reported ongoing per vaginal bleeding following this, with a significant drop in hemoglobin. Contrast enhanced CT $(\mathrm{A}, \mathrm{B})$ demonstrates multiple complex serpentine abnormal vessels in the uterus predominantly on the left side with intense enhancement during arterial phase and show early venous return, consistent with a uterine arteriovenous fistula (AVF). (C) Selective left uterine angiography confirms the AVF, drained by large bilateral gonadal veins. (D) The left uterine artery was embolized using Gelfoam pledgets, with good result. 

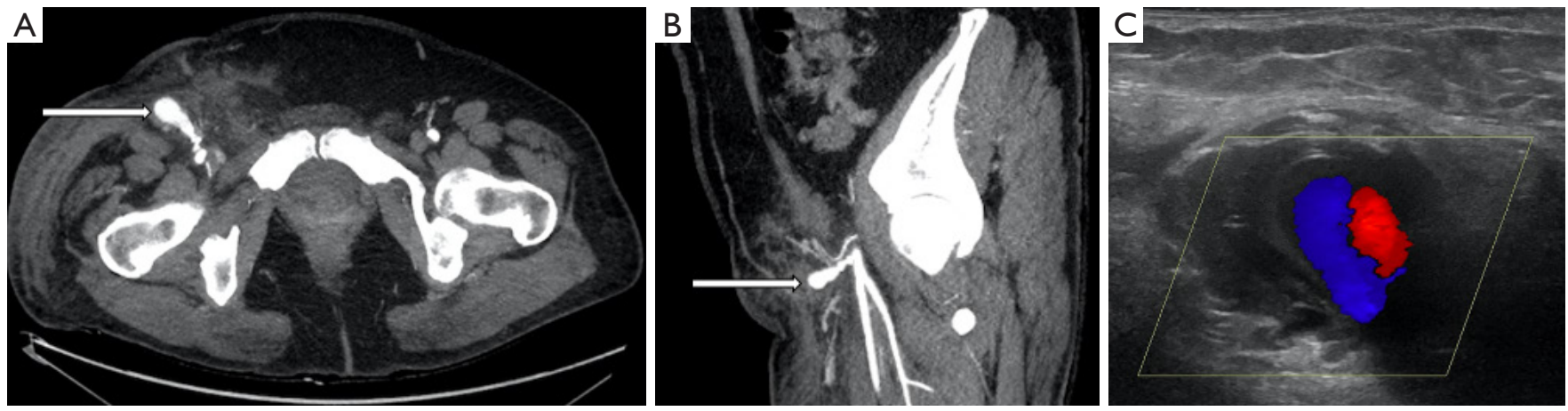

Figure 3 A 48-year-old female who developed a tender pulsatile mass in the right groin one day after coronary angiography using right femoral access. (A,B) Contrast enhanced CT angiography shows a $2.9 \mathrm{~cm}$ narrow necked right femoral artery pseudoaneurysm (long arrow). (C) Turbulent flow and the characteristic "to-and-fro" sign are evident on ultrasound. Four hundred IU of thrombin was injected into the pseudoaneurysm under ultrasound guidance with good result.

Recommendations state that stable patients should have contrast enhanced CT or direct catheter angiography, with or without embolization, whilst unstable patients should proceed directly to theatre for surgical intervention, or in circumstances where endovascular intervention is the preferred management pathway, the patient should be stabilized using pelvic packing, external stabilization or resuscitative endovascular balloon occlusion of the aorta (REBOA) (3) prior to endovascular intervention. Obtaining rapid arterial evaluation and control of bleeding are the priorities during endovascular treatment of an unstable patient. Therefore, one may elect to perform embolization of the internal iliac arteries to prevent exsanguination as there is insufficient time for super selective treatment.

\section{Blunt Trauma}

In the pelvis, blunt trauma is generally as a result of pelvic fractures and can be associated with high morbidity and mortality rates (18). Mortality rates are reported to be approximately $30 \%$ in patients with pelvic fractures who present to the emergency department with clinical signs of shock, primarily due to hemorrhage $(19,20)$. Blood loss can be from exposed cancellous bone, macerated soft tissues, venous and arterial injuries which can be exacerbated by coagulopathy. Blunt trauma can generate shear stresses on vessel walls, avulse a vascular pedicle or cause direct penetrating injury. Arterial injuries such as contusion resulting in intimal defects, subintimal hematomas and occlusion are typical of blunt trauma whilst segmental spasm can be associated with either penetrating or blunt trauma (17).
It has previously been reported that vertical shear fractures most frequently require angiography, followed in frequency by anterior/posterior and lateral compression fractures $(7,21)$. Anterior/posterior pelvic fractures are typically associated with injury to the posterior division of the internal iliac artery, whilst lateral compression fractures more commonly injure the anterior division of the internal iliac artery (22). Mortality remains high in patients with pelvic fractures following trauma due to hemorrhage and difficulty obtaining hemostasis resulting in rapid exsanguination (3). According to Grotz et al., patient's with post traumatic pelvic fracture are generally young with a high injury severity score (23) and it is recommended that patients are treated aggressively to control hemorrhage and sepsis with multidisciplinary team input. This concept is further reiterated by The Society of Interventional Radiology (SIR) (5), Hornez et al. (24) and the World Society of Emergency Surgery (WSES) (3) in their papers on the management of pelvic trauma. The WSES sub classify pelvic injuries into minor (WSES grade I: hemodynamically and mechanically stable), moderate (WSES grade II and III: hemodynamically stable and mechanically unstable) and severe categories (WSES grade IV: hemodynamic instability independent of mechanical status). WSES recommends conservative management for minor injuries and CT with or without embolization for moderate injuries (or laparotomy with or without embolization in certain cases). In the case of severe injury WSES recommends pelvic packing followed by either temporary mechanical embolization, REBOA, or embolization followed by vascular protocol CT for further evaluation.

Blunt trauma may also result in vascular damage without 
an associated fracture such as straddle injuries. This type of injury may result in priapism due to injury to the perineum resulting in arteriovenous or arteriocavernous fistula with partial venous occlusion (7). Embolization of the internal pudendal artery, the terminal branch of the anterior trunk internal iliac artery, is the first line intervention (25) for treatment of high flow priapism following failure of conservative management (26). Of note, the bulbar artery (which supplies the bulb of the urethra, posterior corpus cavernosum and bulbourethral glands) demonstrates normal capillary blush within the bulbar spongiosa. This is an important point to remember when performing penile angiography, as it is unique and similar to the typical appearance of active haemorrhage and should not be mistaken as such. Surgical ligation of the internal pudendal artery may also be performed however, post-surgery impotence is reported in in $10-50 \%$ of cases $(27,28)$.

\section{Anatomy}

Endovascular treatment requires a comprehensive understanding of male and female pelvic arterial and venous anatomy to identify sites of vascular trauma and foresee potential complications of treatment. Considerable variant anatomy may exist and recognizing the presence of aberrant vessels on CT angiography (CTA) and digitally subtracted angiography (DSA) can be crucial to achieving prompt and effective treatment. Failure to identify such variant anatomy may increase the risk of treatment failure and complication (29).

Arterial supply to the pelvis is provided by the internal iliac artery and, to a lesser extent, the external iliac artery. In $60-80 \%$ (30) of cases the internal iliac artery divides into two trunks; anterior and posterior. The anterior trunk supplies many pelvic organs and has the following branches: obturator, umbilical, inferior vesical, vaginal, uterine, middle rectal, internal pudendal and inferior gluteal arteries. The posterior trunk supplies the posterior abdominal wall and pelvis through the iliolumbar, lateral sacral, and superior gluteal arteries. Most traumatic vascular injuries will involve these vessels or their branches.

Important variant arterial supply to be aware of during endovascular procedures include middle rectal, accessory pudendal and persistent sciatic arteries. Middle rectal arteries are present in $30-40 \%$ of the population $(20,31)$, typically arising from a common origin with the prostatic arteries, the prostato-rectal trunk (30). Anastomoses with the superior and inferior rectal arteries are common and may provide a source of non-targeted embolization or recurrent hemorrhage (31).

Accessory pudendal arteries provide the main supply to the penile corpus cavernosum in 3\% (30) and inadvertent embolization raises the risk of erectile and sexual dysfunction (32).

Persistent sciatic arteries are uncommon, with an incidence of $0.03-0.06 \%$ (30), but represent an important normal variant. A persistent embryologic remnant, the persistent sciatic artery can provide aberrant supply to the lower limbs as the femoral system fails to develop $(30,33)$. Inadvertent embolization can lead to critical limb ischemia in such patients (34).

The external iliac artery has been reported to be injured in the setting of pelvic trauma in $3.5-17 \%$ of cases of pelvic trauma (35-37), and carries a relatively high risk of hemodynamically significant hemorrhage, mortality and morbidity $(35,36)$. As such, thorough assessment of the external iliac arteries is vital to successful endovascular management. Of particular importance is the variant collateral pathway known as the Corona Mortis, or Crown of Death. Here, an aberrant obturator artery arises from the deep inferior epigastric artery and joins the external iliac artery (38). The location of the artery which courses over and posterior to the pubic symphysis renders it prone to injury by trauma or during surgical procedures such as hernia repair. Early identification of this vessel is important following trauma as endovascular treatment is required to provide hemostasis (39).

Consideration should also be given in the female pelvis to the origin of the main uterine arteries and supplementary branches arising from the ovarian or contralateral uterine arteries in the setting of hemorrhage and embolization (39). Failure to correctly identify all involved branches in uterine hemorrhage is a strong risk factor for treatment failure (29).

\section{Non-invasive imaging techniques}

Appropriate imaging of pelvic trauma patients is crucial to identify appropriate treatment pathways in a timely manner. Commonly employed imaging modalities include plain film radiography, focused assessment with sonography in trauma (FAST) ultrasound scanning and contrast-enhanced CT.

\section{Plain film}

Plain films provide an appropriate first line for assessing pelvic fractures, however, these can be relatively insensitive, 
particularly in osteoporotic patients (40). Sensitivities of approximately $70 \%$ have been reported with particular insensitivity for posterior fractures on anteroposterior pelvic radiographs. As such, CT is often indicated to assess for radiographically occult fractures $(41,42)$.

\section{FAST}

FAST scanning provides a useful initial emergency department assessment of pelvic trauma and hemorrhage (42) which can identify patients who may require emergency surgical intervention $(42,43)$. The sensitivity of FAST increases with the volume of hemorrhage $(41,44)$ with overall sensitivity for any hemorrhage reported between $50-80 \%(41,45,46)$. In hemodynamically stable patients, a negative FAST scan can reliably exclude the need for endovascular or surgical intervention although a positive study offers poor predictive value of the need for intervention. Given the relatively high false negative rates, some concern has been raised in the setting of negative or equivocal scans linked with higher mortality rates (47). FAST should not delay primary survey and appropriate resuscitation (48). Further limitations of FAST include the inability to identify ongoing bleeding or its source (49). Given the subjective nature of ultrasound, the adequacy of FAST is highly contingent on sonographer skill.

\section{Contrast enhanced CT}

Contrast enhanced computed tomography (CECT) has emerged as a major tool in the investigation of pelvic trauma and vascular injury (49). The pelvic arterial anatomy can be reliably delineated, and bleeding arterial sources identified. The sensitivity and specificity of CECT using a 64 slice multidetector scanner for detecting extravasation, or "blush", are reported at $94 \%$ and $89 \%$, respectively (50). Multiphasic scanning protocols to include non-contrast, arterial and delayed phase sequences can further improve specificity. Following an unenhanced sequence, contrast enhanced CT should be performed with a high flow rate (4-6 mL/s) and approximately $80-120 \mathrm{~mL} / \mathrm{s}$ of intravenous contrast $(49,51)$. Arterial phase imaging should be acquired 25-30 seconds after contrast injection and delayed phase after 60 seconds.

CT examination has the added benefit of providing evaluation of pelvic hematoma, visceral pelvic injury and, fracture delineation (49). Pre-operative evaluation of variant anatomy can also be of value to guide endovascular management. CT can be used to assist decision making and triage of patients who need angiography and embolization from those who do not (50). The identification of extravasation does not alone define the need for endovascular intervention $(50,52,53)$ although higher overall mortality rates have been observed in patients with extravasation (53-55). Other factors to consider when deciding to pursue an endovascular course of treatment include hemodynamic instability, high transfusion needs and a direct relationship between extravasation and pelvic hematoma $(50,52,53)$. Multiple pathways for the assessment and management of pelvic trauma have been suggested (49) with recent guidelines also being published, such as those previously described by WSES (3) and Hornez et al. (24). Departmental consideration should be given to adaptation of such management pathways as rapid, appropriate treatment and multidisciplinary care have been shown to decrease mortality and improve outcomes $(3,8,49)$. Careful assessment of non-vascular structures on CT is also essential in the setting of vascular trauma as small and large bowel, bladder, ureteric, urethral, and gynecologic injuries can coexist, and a coordinated approach is required for optimal patient outcome.

\section{Management}

Emergent management of acute pelvic trauma should focus on the control of hemodynamic instability in the first instance (WSES). Effective cessastion of acute hemorrhage is essential to stabilization of the acutely unwell patient. Aspects of management may include intensive resuscitation, external pelvic binding, REBOA and transcatheter arterial embolization. In the case of pseudoaneurysm, stent placement or thrombin injection are the most common methods of management.

\section{Thrombin Injection}

The options for treatment of a femoral pseudoaneurysm arising secondary to trauma or iatrogenic injury include manual compression in the first instance and if this is unsuccessful, ultrasound guided thrombin injection (Figures 3 and 4). Thrombin is injected into the pseudoaneurysm under ultrasound guidance and resolution of flow to the pseudoaneurysm sac can be assessed at the time of treatment which also determines the volume of thrombin injected (54). This has been shown to have a high success rate, reported between $91 \%$ and $100 \%$, whilst 

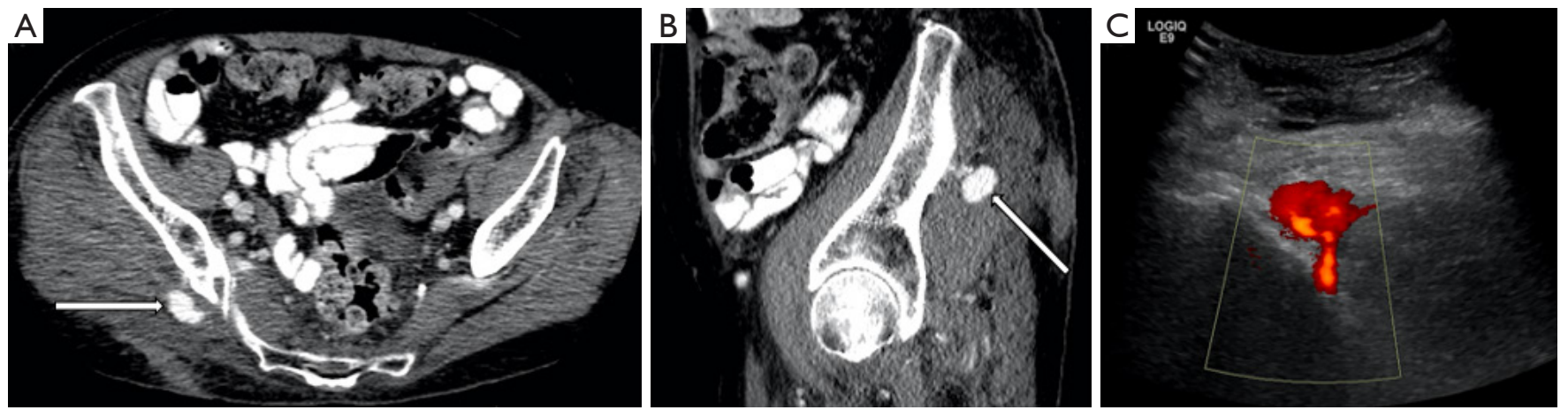

Figure 4 A 75 -year-old female with worsening ecchymosis and acute drop in hemoglobin following a fall. (A,B) Contrast enhanced CT and color doppler ultrasound (C) show a narrow necked pseudoaneurysm arising from the right superior gluteal artery. This was injected under ultrasound guidance with five hundred IU of thrombin with good result.

complications are rare, with embolic events reported in $0.6 \%$ (55). Repeat procedure can be performed with good effect in cases of failure or recanalization (54). Some authors suggest that the size of the aneurysmal neck does not affect the efficacy of treatment, however, in practice thrombin injection of a pseudoaneurysm of the common femoral artery with a wide neck risks embolic complications without temporary covering balloon occlusion of the parent vessel (56). Alternatively, surgery is often considered for the treatment of such lesions since stent placement across a flexure has a higher incidence of subsequent stent fracture.

\section{REBOA}

Recent research in the emergency room management of pelvic hemorrhage has focused on the resurgent use of REBOA. REBOA involves placement of an occlusive balloon within the aorta via femoral arterial access with the aim of reducing direct pelvic vascular inflow as a bridge to definitive surgical or endovascular treatment $(57,58)$. Current consensus guidelines (WSES) highlight the lack of high-grade evidence supporting its use but suggest careful use in the setting of infradiaphragmatic hemorrhage which is refractory to resuscitation as a potential indication (57). Sites of balloon placement are defined within three anatomical zones. Abdominal or retroperitoneal hemorrhage requires more proximal inflation within the distal thoracic aorta (Zone 1), with isolated pelvic hemorrhage being amenable to more distal inflation with the distal abdominal aorta (Zone 3) (57).

Conflicting evidence exists on the mortality and morbidity benefits of REBOA with some studies suggesting improved survival to discharge, improved systolic blood pressure and decrease in mortality (59-61) with others suggesting an increased mortality rate with its' usage and urging caution $(48,62)$. Complications of the procedure can be devastating with potentially increased mortality, spinal cord ischemia, reperfusion injury, multiorgan failure and vascular injuries related to access and placement (57). Overall, current guidelines and evidence would suggest the use of REBOA only in the hands of trained and experienced operators with careful thought given to patient selection and balloon siting. Further evidence and analysis are necessary to precisely define optimal indications for use to minimize morbidity and mortality.

\section{Transcatheter arterial embolization}

Transcatheter embolization of arterial hemorrhage has emerged as a mainstay in the management of acute pelvic trauma with efficacy rates of $81-100 \%$ (63). A prospective study of 100 patients showed embolization being $95 \%$ effective and safe in $94 \%$ of both stable and unstable patients with abdominal and pelvic visceral injury (64). As discussed, multiple guidelines and pathways, most recently WSES, have been designed with the aim of streamlining the management of patients with pelvic trauma. A decision to proceed with transcatheter embolization should be multifactorial. WSES guidelines highlight the role of CECT in establishing the need for embolization with the presence of contrast extravasation and pelvic hematoma being the most important predictive signs of the need for embolization (WSES) (Figure 5). The individual pattern of pelvic fractures may be useful for identifying the source of bleeding (18). Higher severity fractures, typically open ring and anterior-posterior compression varieties, are associated 

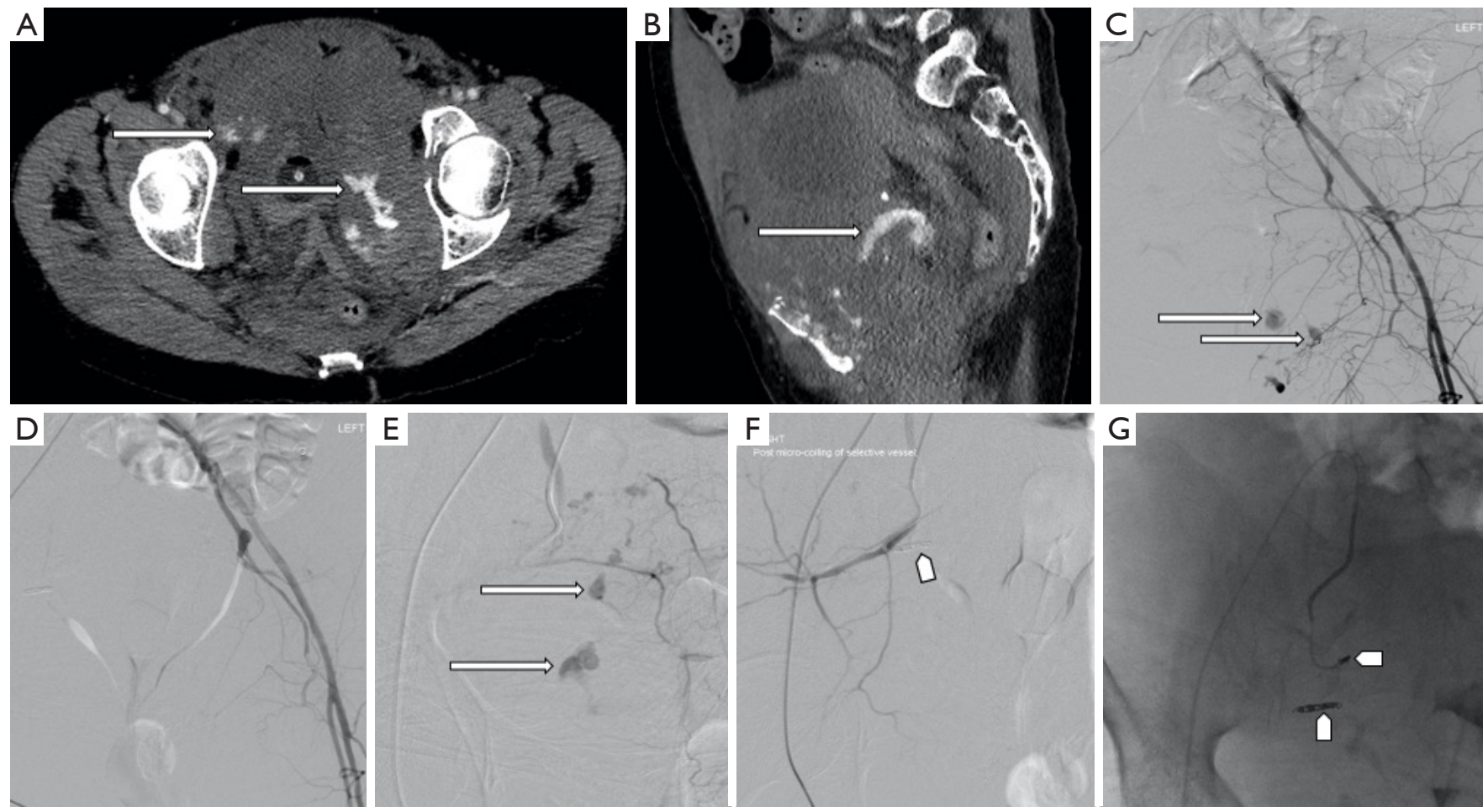

Figure 5 A 65-year-old female who suffered low velocity crush injuries between a car and wall. (A,B) Contrast enhanced CT demonstrates complex pelvic fractures and multiple sites of active hemorrhage (long arrows). (C) The patient underwent selective angiography of bilateral internal iliac arteries confirming this. (D) The posterior trunk of the left internal iliac artery was embolized using Gelfoam. (E,F,G) Multiple sites of hemorrhage persisted. The posterior trunk of the right internal iliac artery was embolized using microcoils, however, the entire right internal iliac artery was eventually embolized using Gelfoam to achieve hemostasis.

with a greater need for hemorrhage control but do not alone predict the need for embolization $(65,66)$ (Figures 6 and 7). As previously mentioned, a practical consideration for endovascular intervention is the availability of specialized staff and equipment. There should either be a dedicated interventional radiology team on standby, with round the clock access to an interventional suite or portable equipment in a large operating theatre or hybrid suite with theatre staff being familiar with equipment in order to allow for timely patient treatment.

\section{Angiography}

Angiography provides diagnostic and therapeutic options for treatment. Antecedent CT and fracture patterns should inform angiography technique. Note should be made of any co-existing thoracic or abdominal injuries which may require further evaluation. Evidence of arterial hemorrhage or uncontrolled retroperitoneal hemorrhage on CT is an indication for angiography. Injuries to the external iliac artery often entail intimal tear and vasospasm. Internal iliac injuries most commonly affect the superior gluteal artery and less commonly the internal pudendal and obturator arteries. Other potential sources of bleeding include the lumbar, inferior epigastric and circumflex iliac arteries. Bladder drainage using a foley catheter helps remove excreted contrast but not at the expense of wasted time in emergent cases.

Arterial access should be obtained in the common femoral artery on the contralateral side in the case of hemipelvis injury. If use of this location is not possible then the left radial, brachial or axillary approach can be used (67). Either a micropuncture (Cook Medical, Bloomington, IN) or 18-guage needle can be used and this can be performed under ultrasound guidance or direct palpation of the vessel using standard Seldinger technique. If using a micropuncture needle, a 0.018/40 nitinol wire with pallidium tip should be inserted under fluoroscopic guidance and the needle exchanged for a $5-\mathrm{F}-10 \mathrm{~cm}$ vascular sheath (Terumo Interventional Systems, Somerset, 

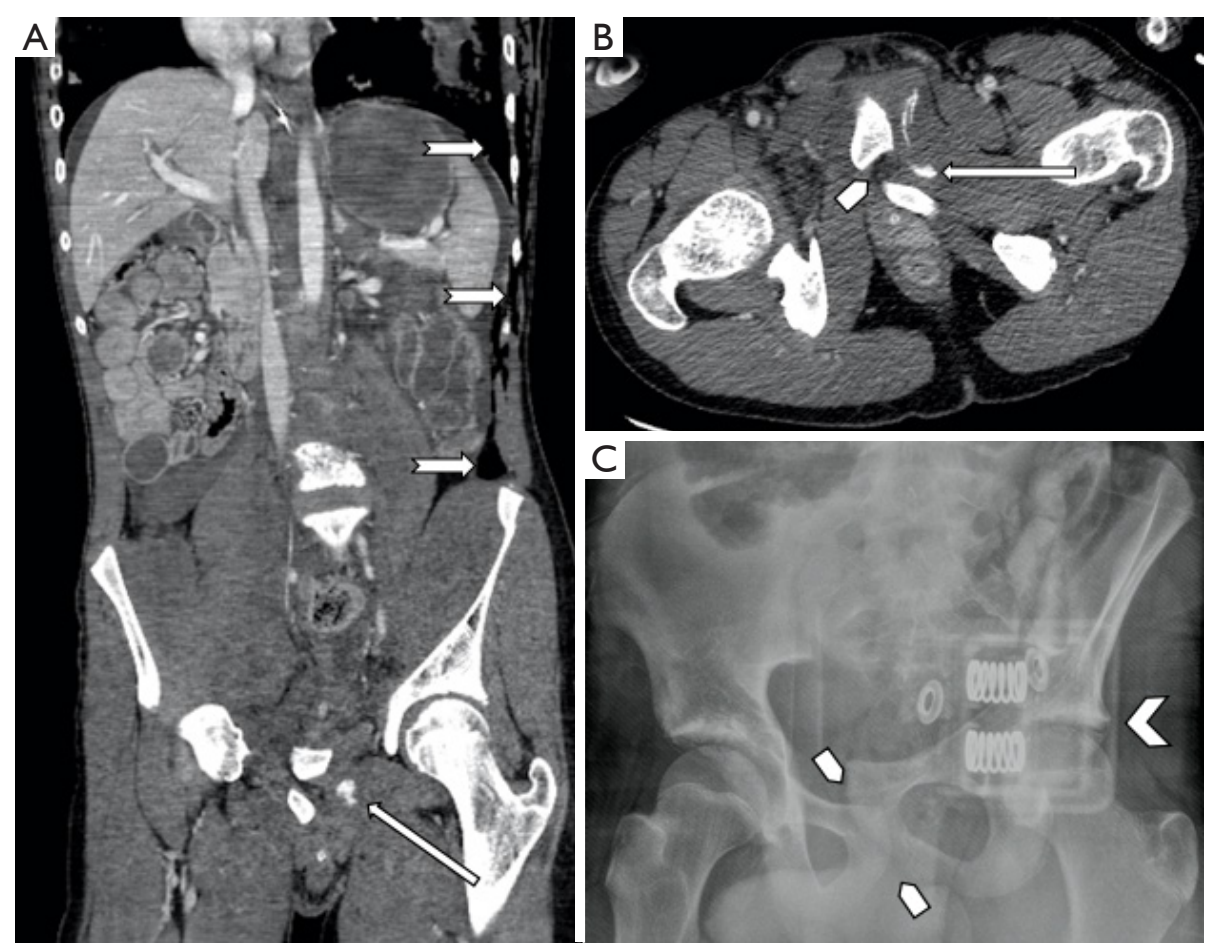

Figure 6 A 15-year-old male who suffered multiple complex crush injuries from a farming accident. (A,B) Contrast enhanced CT shows active hemorrhage in the left hemipelvis (long arrow). (B,C) Complete disruption of the pubic symphysis (pentagon arrow) with complete posterosuperior dislocation of the left pubic rami. (C) Note the presence of a pelvic binder (arrowhead) on plain film. (A) There were multiple other injuries including unstable spinal injuries, bilateral pulmonary lacerations, pneumohemothoraces and pneumoperitoneum (notched arrows). This patient responded well to conservative management and therefore did not require endovascular intervention as per multidisciplinary team consensus.
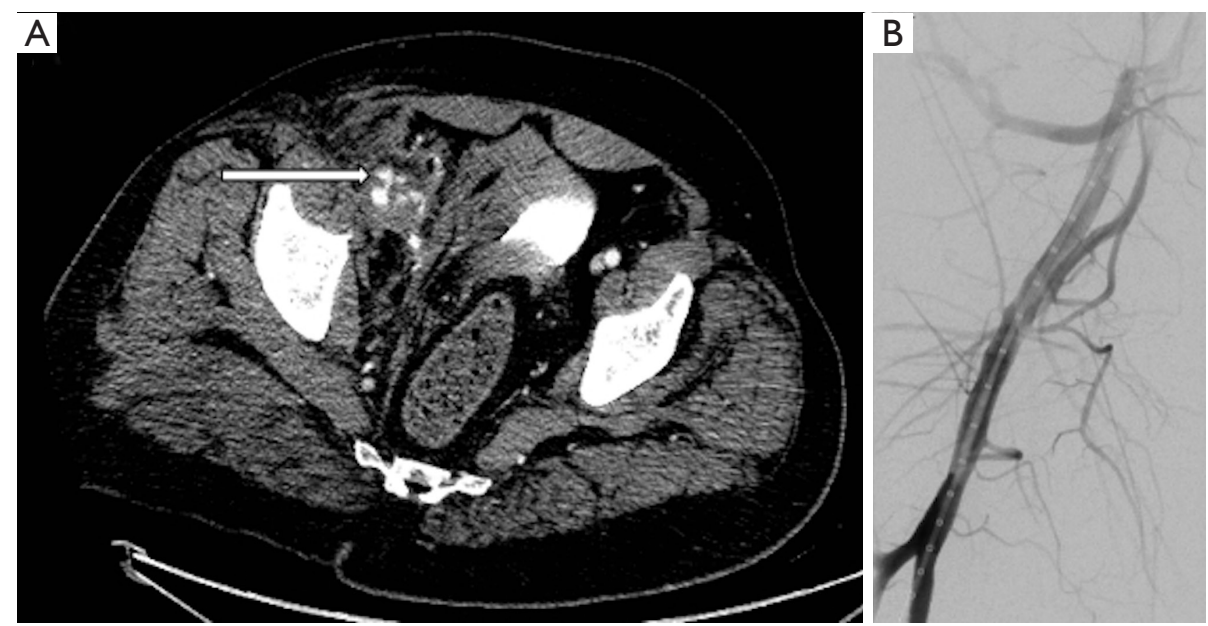

Figure 7 A 58-year-old man who developed right lower abdominal pain following coronary angiography. A micropuncture needle was used to access the vessel, however the access point was repositioned as was deemed too high. (A) CT angiography demonstrated active hemorrhage from the right common femoral artery (arrow). The patient was anticoagulated, which was subsequently reversed and was initially managed conservatively. (B) Subsequent catheter angiography showed no site of active hemorrhage. 
$\mathrm{NJ})$.

A flush catheter such as a $5 \mathrm{~F}$ pigtail or Omni Flush catheter (AngioDynamics, Queensbury, NY) should be advanced over a non-traumatic floppy wire of choice such as a Bentson (Cook Medical, Bloomington, IN), glide or J wire with initial nonselective angiography being performed to gain visualization of the arterial supply of the pelvis and lumbar regions, anatomical variants, obvious points of extravasation or occlusion and arteries adjacent to the region of injury. An aortogram should initially be performed to identify proximal large vessel injury above the aortic bifurcation using a pump injector at a rate of $15-20 \mathrm{~mL} /$ second using $30-40 \mathrm{~mL}$ of intravenous contrast medium. This can be imaged using conventional fluoroscopy (either continuous or high pulse rate fluoroscopy), however digitally subtracted angiography is the imaging modality of choice and a roadmap may also be created to aid navigation. Posterior and anterior oblique projection angiography of the internal and external iliac arteries should then be performed and supplemented by selective angiography as required especially when nonselective imaging fails to show extravasation. Multiple projections help assessment and overcome overlying fixation devices and other sources of artefact (68).

Once the anatomy and/or site of injury has been established, a guidewire should be advanced over the bifurcation to the side of injury. If difficulty is encountered at this point, the Bentson or J wire may be exchanged for a 0.035 floppy or stiff angled glide wire (Terumo Interventional Systems). If difficulty persists a 4 or $5 \mathrm{~F}$ Cobra C2 Glidecath (Terumo Interventional Systems), Waltmann loop, or reverse curve catheter may aid in crossing the bifurcation (69). Even if no point of hemorrhage is identified on flush angiography, dedicated selective angiography should be performed of bilateral internal iliac arteries (67) as these have been shown to be the most common site of hemorrhage in pelvic trauma (70).

When performing pelvic or vascular intervention from either the contralateral side or distal site it is important that a wide range of equipment is available such as exchange length wires $(>260 \mathrm{~cm})$, long $(90-120 \mathrm{~cm})$, or wide $(7-10 \mathrm{~F})$ long vascular sheaths, which can provide a stable platform facilitating treatment and reducing the risks of non-target embolization.

Depending on the type of vessel injury that has occurred, there are a multitude of possible agents and devices that can be used in the management of pelvic trauma. Often the method of treatment is based on local practices and experience as there is little evidence for one method or device over another. For example, stents are considered suitable for treatment of intimal injury resulting in a dissection flap or an incomplete transection which can restore luminal continuity in a major pelvic vessel such as the iliac arteries (71). Stents are generally oversized by $10-20 \%$ of the vessel diameter to ensure good opposition with the intimal walls, reducing the risk of endoleak (72). Stenting of vessels across a flexure is generally avoided due to difficulty obtaining opposition with the vessel wall and risk of future stent fracture (67).

In the case of a single bleeding vessel selective embolization is often the best option, however the approach to this is often subjective and multifactorial as there are a wide variety of embolization agents and techniques available. Autologous blood clot was initially used as an embolization agent in the setting of trauma; however, these are quickly hemolyzed and considered outdated. Temporary embolization agents have been developed, which are widely available, simple to use and relatively inexpensive, such as Gelfoam pledgets or slurry (Upjohn, Kalamazoo, MI), which are the most commonly used. Pledgets should be cut to suit the size of the target vessel; usually $1 \mathrm{~mm}$ and larger suspended in contrast and delivered incrementally using a Luer-lock syringe. Long strips help achieve large vessel embolization. Gelfoam is degraded over approximately 7-21 days and are generally suitable for microcatheter use. These should be injected slowly, and injection should cease prior to complete occlusion in order to ensure adequate embolization of the desired vessel, whilst preventing reflux proximally and the inadvertent occlusion of nontargeted vessels (Figure 8). If selective embolization is unsuccessful, a nonselective embolization may be performed to embolize the internal iliac vessels proximally ensuring complete embolization of vessels within the pelvis to obtain hemostasis (67).

Other devices widely used in the management of pelvic hemorrhage, include coils and vascular Amplatzer plugs (AGA Medical Corporation, Plymouth, MN). The Amplatzer plug is a self-expandable nitinol occlusion device, which is considered to be permanent. Typically, coils are impregnated with thrombogenic materials (e.g., wool, nylon fibers, silk and polyester) which activate the coagulation cascade resulting in thrombus formation and subsequent vessel occlusion. Multiple coils may be inserted to create a scaffold, the interstices of which can be subsequently filled with smaller coils, however these methods prevent future distal intervention at a later point (73) 

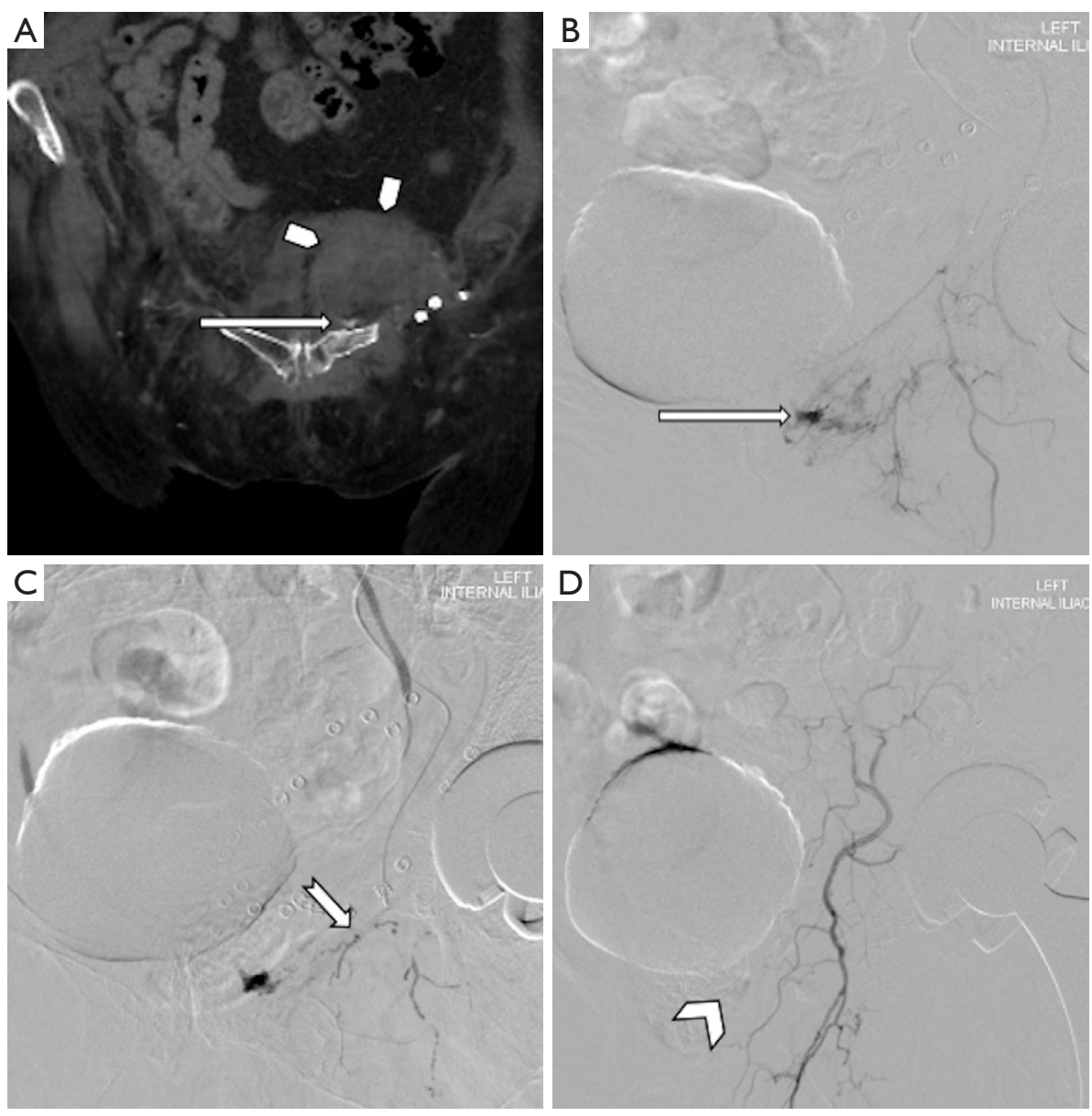

Figure 8 An 81-year-old female with worsening mobility and hemoglobin drop following a fall. Contrast enhanced CT (A) shows a large hematoma (pentagon arrows) in the left hemipelvis with a focal point of active hemorrhage (long arrow) from the left obturator artery was demonstrated on both CT (A) and subsequent direct catheter angiography (B,C,D). The vessel was successfully embolized (arrowhead, D) with 25\% N-butyl cyanoacrylate (nBCA) (tapered arrow, C).

as can be required if collateral vessels lead to repeated hemorrhage.

In the case of a hemodynamically unstable patient, timely resuscitation and hemostasis is of critical importance. In such circumstances, it is important that all management options are availed of in order to optimize the patient's hemodynamic status, including the initial practical conservative methods such as the application of a pelvic binder, presence of highly trained emergency/anesthetic physicians and activation of major blood transfusion protocols to ensure adequate volume resuscitation. With regard to the endovascular approach to the unstable patient, superselective angiography is not deemed appropriate as it can be time consuming and delay hemostasis. Bland embolization with Gelfoam of the anterior/posterior trunks of the internal iliac artery or hypogastric arteries in a pulsed manner is often advocated (74). This is a life-saving procedure and although there is a risk of necrosis to the pelvic organs it is generally well tolerated since the bladder, rectum and pelvic soft tissues have ample collateral blood supply (75). Temporary agents such as Gelfoam should be used in an effort to reduce long term morbidity (76).

Complications encountered during and after pelvic arterial embolization can relate to the arterial access point, typically in the femoral artery, or the procedure itself. Access site complications include bleeding and hematoma formation, pseudoaneurysms and arteriovenous fistulae. The incidence of these complications varies. Pseudoaneurysms are reported following approximately $1 \%$ and arteriovenous fistulae in $0.2-2.1 \%$ of vascular procedures (76). Significant 
retroperitoneal hemorrhage is generally related to inappropriately high arterial access above the inferior border of the inferior epigastric artery $(77,78)$. Access site complications may be significantly minimized with the use of fluoroscopic guidance (79) and further still with ultrasound guided puncture $(80,81)$.

Contrast induced nephropathy (CIN) may occur in up to $20 \%$ of cases (42) however concerns regarding renal function should not delay what is a time critical and potentially life-saving treatment in the setting of pelvic trauma. The majority of complications relating to arterial embolization itself occur either inadvertently as a consequence of nontarget embolization or simply as an unavoidable result of necessary large vessel or bilateral embolization. Ischemia or necrosis may involve the gluteal muscles, femoral head, lower limb or pelvic viscera (42). Careful consideration of necessary embolization targets and patient anatomy can help to minimize these complications. The nature and results of an intervention needs to be discussed and carefully documented following IR procedures. Referring physicians need to be aware of potential complications which could occur in order to ensure early assessment for detection and treatment.

\section{Conclusion}

Pelvic trauma is a highly variable and complex pathology, with potentially life-threatening catastrophic outcomes. There are a wide variety of vascular injuries which can occur in the setting of pelvic trauma and many management options are available, with endovascular management ever growing in importance. Initial management should focus on patient stabilization using a multidisciplinary approach, followed by cross sectional imaging where indicated. If active hemorrhage is suspected, confirmed or a patient in extremes where direct surgical intervention is not practical, endovascular intervention can provide rapid access and control of hemorrhage leading to better patient outcomes.

\section{Acknowledgments}

Funding: None.

\section{Footnote}

Provenance and Peer Review: This article was commissioned by the Guest Editor (Dr. Keith Bertram Quencer) for the series "Endovascular interventions in trauma" published in Annals of Translational Medicine. The article has undergone external peer review.

Conflicts of Interest: All authors have completed the ICMJE uniform disclosure form (available at http://dx.doi. org/10.21037/atm-20-4591). The series "Endovascular interventions in trauma" was commissioned by the editorial office without any funding or sponsorship. The authors have no other conflicts of interest to declare.

Ethical Statement: The authors are accountable for all aspects of the work in ensuring that questions related to the accuracy or integrity of any part of the work are appropriately investigated and resolved.

Open Access Statement: This is an Open Access article distributed in accordance with the Creative Commons Attribution-NonCommercial-NoDerivs 4.0 International License (CC BY-NC-ND 4.0), which permits the noncommercial replication and distribution of the article with the strict proviso that no changes or edits are made and the original work is properly cited (including links to both the formal publication through the relevant DOI and the license). See: https://creativecommons.org/licenses/by-nc-nd/4.0/.

\section{References}

1. Magnone S, Coccolini F, Manfredi R, et al. Management of hemodynamically unstable pelvic trauma: results of the first Italian consensus conference (cooperative guidelines of the Italian Society of Surgery, the Italian Association of Hospital Surgeons, the Multi-specialist Italian Society of Young Surgeons, the Italian Society of Emergency Surgery and Trauma, the Italian Society of Anesthesia, Analgesia, Resuscitation and Intensive Care, the Italian Society of Orthopaedics and Traumatology, the Italian Society of Emergency Medicine, the Italian Society of Medical Radiology -Section of Vascular and Interventional Radiology- and the World Society of Emergency Surgery). World J Emerg Surg 2014;9:18.

2. Arvieux C, Thony F, Broux C, et al. Current management of severe pelvic and perineal trauma. J Visc Surg 2012;149:e227-38.

3. Coccolini F, Stahel PF, Montori G, et al. Pelvic trauma: WSES classification and guidelines. World J Emerg Surg 2017;12:5.

4. Jacks R, Degiannis E. Endovascular Therapy And Controversies In The Management Of Vascular Trauma. 
Scand J Surg 2014;103:149-55.

5. Padia SA, Ingraham CR, Moriarty JM, et al. Society of Interventional Radiology Position Statement on Endovascular Intervention for Trauma. J Vasc Interv Radiol 2020;31:363-369.e2.

6. Rich NM, Rhee P. An historical tour of vascular injury management: From its inception to the new millennium. Surg Clin North Am 2001;81:1199-215.

7. Nicholson AA. Vascular Radiology in Trauma. Cardiovasc Intervent Radiol 2004;27:105-20.

8. Gilbert F, Schneemann C, Scholz CJ, et al. Clinical implications of fracture-associated vascular damage in extremity and pelvic trauma 11 Medical and Health Sciences 1103 Clinical Sciences. BMC Musculoskelet Disord 2018;19:404.

9. Pryor A, Mann WJ, Bates AT. Complications of laparoscopic surgery. UpToDate. 2020.

10. Simforoosh N, Basiri A, Ziaee SAM, et al. Major vascular injury in laparoscopic urology. JSLS 2014;18:e2014.00283.

11. Krishnakumar S, Tambe P. Entry complications in laparoscopic surgery. J Gynecol Endosc Surg 2009;1:4-11.

12. Parker WH, Wagner WH. Management of hemorrhage in gynecologic surgery. Obstet Gynecol Clin North Am 2010;37:427-36.

13. Kamaya A, Petrovitch I, Chen B, et al. Retained Products of Conception. J Ultrasound Med 2009;28:1031-41.

14. Kimura Y, Osuga K, Nagai K, et al. The efficacy of uterine artery embolization with gelatin sponge for retained products of conception with bleeding and future pregnancy outcomes. CVIR Endovasc 2020;3:13.

15. Gipson MG, Smith MT. Endovascular therapies for primary postpartum hemorrhage: Techniques and outcomes. Semin Intervent Radiol 2013;30:333-9.

16. García Blanco VE, Ruales Romero AM, Galera Martínez MC, et al. Ruptured Anastomotic Pseudoaneurysm of the Common Femoral Artery After Wrongful Diagnosis of Inguinal Hernia. Cir Esp 2018;96:459-61.

17. Mavrogenis AF, Panagopoulos GN, Kokkalis ZT, et al. Vascular injury in orthopedic trauma. Orthopedics 2016;39:249-59.

18. Hussami M, Grabherr S, Meuli RA, et al. Severe pelvic injury: vascular lesions detected by ante- and post-mortem contrast medium-enhanced CT and associations with pelvic fractures. Int J Legal Med 2017;131:731-8.

19. Costantini TW, Coimbra R, Holcomb JB, et al. Current management of hemorrhage from severe pelvic fractures. J Trauma Acute Care Surg 2016;80:717-23.

20. Ooi CK, Goh HK, Tay SY, et al. Patients with pelvic fracture: What factors are associated with mortality? Int J Emerg Med 2010;3:299-304.

21. Asensio JA, Trunkey D. Current Therapy of Trauma and Surgical Critical Care. Elsevier 2008.

22. Sheridan MK, Blackmore CC, Linnau KF, et al. Can CT predict the source of arterial hemorrhage in patients with pelvic fractures? Emerg Radiol 2002;9:188-94.

23. Grotz MRW, Allami MK, Harwood P, et al. Open pelvic fractures: Epidemiology, current concepts of management and outcome. Injury 2005;36:1-13.

24. Hornez E, Monchal T, Boddaert G, et al. Penetrating pelvic trauma: Initial assessment and surgical management in emergency. J Visc Surg 2016;153:79-90.

25. Fratezi AC, Martins VM, Pereira Porta RM, et al. Endovascular therapy for priapism secondary to perineal trauma. J Trauma 2001;50:581-4.

26. Khera PS, Garg PK, Pandey H, et al. Post-traumatic highflow priapism: Uncommon presentation with endovascular management. BMJ Case Reports 2019;12:229299.

27. O'Sullivan P, Browne R, McEniff N, et al. Treatment of "high-flow" priapism with superselective transcatheter embolization: A useful alternative to surgery. Cardiovasc Intervent Radiol 2006;29:198-201.

28. Ji MX, He NS, Wang P, et al. Use of selective embolization of the bilateral cavernous arteries for posttraumatic arterial priapism. J Urol 1994;151:1641-2.

29. Bros S, Chabrot P, Kastler A, et al. Recurrent bleeding within 24 hours after uterine artery embolization for severe postpartum hemorrhage: Are there predictive factors? Cardiovasc Intervent Radiol 2012;35:508-14.

30. Bilhim T, Pereira JA, Fernandes L, et al. Angiographic Anatomy of the Male Pelvic Arteries. AJR Am J Roentgenol 2014;203:W373-82.

31. Bilhim T, Pereira JA, Tinto HR, et al. Middle rectal artery: Myth or reality? Retrospective study with CT angiography and digital subtraction angiography. Surg Radiol Anat 2013;35:517-22.

32. Kably I, Dupaix R. Prostatic Artery Embolization and the Accessory Pudendal Artery. J Vasc Interv Radiol 2016;27:1266-8.

33. van Hooft IM, Zeebregts CJ, van Sterkenburg SMM, et al. The Persistent Sciatic Artery. Eur J Vasc Endovasc Surg 2009;37:585-91.

34. Huang X, Tang Y, Xu G, et al. Loss of Limb by Inadvertent Embolization of the Persistent Sciatic Artery. Vasc Endovascular Surg 2016;50:60-2.

35. Cestero RF, Plurad D, Green D, et al. Iliac Artery Injuries and Pelvic Fractures: A National Trauma Database 
Analysis of Associated Injuries and Outcomes. J Trauma 2009;67:715-8.

36. Tanizaki S, Maeda S, Ishida H, et al. Clinical characteristics of external iliac artery branch injury in pelvic trauma. Am J Emerg Med 2017;35:1636-8.

37. Johnson GE, Sandstrom CK, Kogut MJ, et al. Frequency of external iliac artery branch injury in blunt trauma: Improved detection with selective external iliac angiography. J Vasc Interv Radiol 2013;24:363-9.

38. Sanna B, Henry BM, Vikse J, et al. The prevalence and morphology of the corona mortis (Crown of death): A meta-analysis with implications in abdominal wall and pelvic surgery. Injury 2018;49:302-8.

39. Herskowitz M, Walsh J, Lilly M, et al. Importance of Both Internal and External Iliac Artery Interrogation in Pelvic Trauma as Evidenced by Hemorrhage from Bilateral Corona Mortis with Unilateral Aberrant Origin off the External Iliac Artery. Case Rep Radiol 2019;2019:6734816.

40. Ross AB, Lee KS, Chang EY, et al. ACR Appropriateness Criteria ${ }^{\circledR}$ Acute Hip Pain-Suspected Fracture. J Am Coll Radiol 2019;16:S18-25.

41. Eggenberger E, Hildebrand G, Vang S, et al. Use of CT Vs. MRI for Diagnosis of Hip or Pelvic Fractures in Elderly Patients After Low Energy Trauma. Iowa Orthop J 2019;39:179-83.

42. Christian NT, Burlew CC, Moore EE, et al. The focused abdominal sonography for trauma examination can reliably identify patients with significant intra-abdominal hemorrhage in life-threatening pelvic fractures. J Trauma Acute Care Surg 2018;84:924-8.

43. Wijffels DJ, Verbeek DO, Ponsen KJ, et al. Imaging and Endovascular Treatment of Bleeding Pelvic Fractures: Review Article. Cardiovasc Intervent Radiol 2019;42:10-8.

44. Verbeek DOF, Zijlstra IAJ, Van Der Leij C, et al. The utility of FAST for initial abdominal screening of major pelvic fracture patients. World J Surg 2014;38:1719-25.

45. Do WS, Chang R, Fox EE, et al. Too fast, or not fast enough? The FAST exam in patients with non-compressible torso hemorrhage. Am J Surg 2019;217:882-6.

46. Tayal VS, Nielsen A, Jones AE, et al. Accuracy of Trauma Ultrasound in Major Pelvic Injury. J Trauma 2006;61:1453-7.

47. Kleinman J, Strumwasser A, Rosen D, et al. The dangers of equivocal FAST in trauma resuscitation. Am Surg 2017;83:1127-31.

48. Kleinman J, Inaba K, Pott E, et al. Early fast examinations during resuscitation may compromise trauma outcomes. Am Surg 2018;84:1705-9.
49. Bent C, Chicklore S, Newton A, et al. Do emergency physicians and radiologists reliably interpret pelvic radiographs obtained as part of a trauma series? Emerg Med J 2013;30:106-11.

50. Ramin S, Hermida M, Millet I, et al. Limits of intravascular contrast extravasation on computed tomography scan to define the need for pelvic angioembolization in pelvic blunt trauma. J Trauma Acute Care Surg 2018;85:527-35.

51. Pinto A, Niola R, Tortora G, et al. Role of multidetectorrow CT in assessing the source of arterial haemorrhage in patients with pelvic vascular trauma. Comparison with angiography. Radiol Med 2010;115:648-67.

52. Michailidou M, Velmahos GC, van der Wilden G, et al. "Blush" on trauma computed tomography. J Trauma Acute Care Surg 2012;73:580-4.

53. Juern JS, Milia D, Codner P, et al. Clinical significance of computed tomography contrast extravasation in blunt trauma patients with a pelvic fracture. J Trauma Acute Care Surg 2017;82:138-40.

54. Jargiełł T, Sobsty J, wiatłowski Ł, et al. Ultrasoundguided thrombin injection in the management of pseudoaneurysm after percutaneous arterial access. J Ultrason 2018;18:85-9.

55. Kontopodis N, Tsetis D, Tavlas E, et al. Ultrasound Guided Compression Versus Ultrasound Guided Thrombin Injection for the Treatment of PostCatheterization Femoral Pseudoaneurysms: Systematic Review and Meta-Analysis of Comparative Studies. Eur J Vasc Endovasc Surg 2016;51:815-23.

56. Yang EY, Tabbara MM, Sanchez PG, et al. Comparison of Ultrasound-Guided Thrombin Injection of Iatrogenic Pseudoaneurysms Based on Neck Dimension. Ann Vasc Surg 2018;47:121-7.

57. Bulger EM, Perina DG, Qasim Z, et al. Clinical use of resuscitative endovascular balloon occlusion of the aorta (REBOA) in civilian trauma systems in the USA, 2019: A joint statement from the American College of Surgeons Committee on Trauma, the American College of Emergency Physicians, the N. Trauma Surg Acute Care Open 2019;4:376.

58. Coccolini F, Ceresoli M, McGreevy DT, et al. Aortic balloon occlusion (REBOA) in pelvic ring injuries: preliminary results of the ABO Trauma Registry. Updates Surg 2020;72:527-36.

59. Yamamoto R, Cestero RF, Suzuki M, et al. Resuscitative endovascular balloon occlusion of the aorta (REBOA) is associated with improved survival in severely injured patients: A propensity score matching analysis. Am J Surg 
2019;218:1162-8.

60. Manzano Nunez R, Naranjo MP, Foianini E, et al. A metaanalysis of resuscitative endovascular balloon occlusion of the aorta (REBOA) or open aortic cross-clamping by resuscitative thoracotomy in non-compressible torso hemorrhage patients. World J Emerg Surg 2017;12:30.

61. Petrone P, Pérez-Jiménez A, Rodríguez-Perdomo M, et al. Resuscitative endovascular balloon occlusion of the aorta (REBOA) in the management of trauma patients: A systematic literature review. Am Surg 2019;85:654-62.

62. Joseph B, Zeeshan M, Sakran J V., et al. Nationwide Analysis of Resuscitative Endovascular Balloon Occlusion of the Aorta in Civilian Trauma. JAMA Surg 2019;154:500-8.

63. Papakostidis C, Kanakaris N, Dimitriou R, et al. The role of arterial embolization in controlling pelvic fracture haemorrhage: A systematic review of the literature. Eur J Radiol 2012;81:897-904.

64. Velmahos GC, Toutouzas KG, Vassiliu P, et al. A prosupective study on the safety and efficacy of angiographic embolization for pelvic and visceral injuries. J Trauma 2002;53:303-8.

65. Kim MJ, Lee JG, Lee SH. Factors predicting the need for hemorrhage control intervention in patients with blunt pelvic trauma: A retrospective study. BMC Surg 2018;18:101.

66. Costantini TW, Coimbra R, Holcomb JB, et al. Pelvic fracture pattern predicts the need for hemorrhage control intervention-Results of an AAST multi-institutional study. J Trauma Acute Care Surg 2017;82:1030-8.

67. Broadwell SR, Ray CE. Transcatheter embolization in pelvic trauma. Semin Intervent Radiol 2004;21:23-35.

68. Rane N, Imam A, Foley P, et al. Pelvic digital subtraction catheter angiography - Are routine oblique projections necessary? Eur J Radiol 2011;77:182-4.

69. Kevin BY, Herman C. Crossing the Challenging Aortic Bifurcation. Endovasc Today 2012;(January):45-9.

70. Vaidya R, Waldron J, Scott A, et al. Angiography and

Cite this article as: Weir A, Kennedy $\mathrm{P}$, Joyce S, Ryan D, Spence L, McEntee M, Maher M, O'Connor O. Endovascular management of pelvic trauma. Ann Transl Med 2021;9(14):1196. doi: 10.21037/atm-20-4591
Embolization in the Management of Bleeding Pelvic Fractures. J Am Acad Orthop Surg 2018;26:e68-e76.

71. O'Connor O, Brady A, Harte P. Percutaneous management of a traumatic arteriovenous fistula. Ir Med J 2008;101:58-9.

72. Johnson CA. Endovascular management of peripheral vascular trauma. Semin Intervent Radiol 2010;27:38-43.

73. Awwad A, Dhillon PS, Ramjas G, et al. Trans-arterial embolisation (TAE) in haemorrhagic pelvic injury: review of management and mid-term outcome of a major trauma centre. CVIR Endovasc 2018;1:32.

74. Lopera JE. Embolization in trauma: Principles and techniques. Semin Intervent Radiol 2010;27:14-28.

75. Hare WS, Holland CJ. Paresis following internal iliac artery embolization. Radiology 1983;146:47-51.

76. Abrams HL, Baum S, Pentecost MJ. Abrams' angiography: interventional radiology. 2nd ed. Lippincott Williams \& Wilkins; 2006; 1264 p.

77. Bhatty S, Cooke R, Shetty R, et al. Femoral vascular access-site complications in the cardiac catheterization laboratory: Diagnosis and management. Intervent Cardiol 2011;3:503-14.

78. Pitta SR, Prasad A, Kumar G, et al. Location of femoral artery access and correlation with vascular complications. Catheter Cardiovasc Interv 2011;78:294-9.

79. Fairley SL, Lucking AJ, McEntegart M, et al. Routine Use of Fluoroscopic-Guided Femoral Arterial Puncture to Minimise Vascular Complication Rates in CTO Intervention: Multi-centre UK Experience. Heart Lung Circ 2016;25:1203-9.

80. Stone P, Campbell J, Thompson S, et al. A prospective, randomized study comparing ultrasound versus fluoroscopic guided femoral arterial access in noncardiac vascular patients. J Vasc Surg 2020;72:259-67.

81. Sobolev M, Slovut DP, Chang AL, et al. Ultrasoundguided catheterization of the femoral artery: A systematic review and meta-analysis of randomized controlled trials. J Invasive Cardiol 2015;27:318-23. 